\title{
Stochastically excited oscillations in the upper main sequence
}

\author{
Victoria Antoci \\ Stellar Astrophysics Centre, Department of Physics and Astronomy, Aarhus University \\ Ny Munkegade 120, DK-8000 Aarhus C, Denmark \\ email: antoci@phys.au.dk
}

\begin{abstract}
Convective envelopes in stars on the main sequence are usually connected only with stars of spectral types F5 or later. However, observations as well as theory indicate that the convective outer layers in hotter stars, despite being shallow, are still effective and turbulent enough to stochastically excite oscillations. Because of the low amplitudes, exploring stochastically excited pulsations became possible only with space missions such as Kepler and CoRoT. Here I review the recent results and discuss among others, pulsators such as $\delta$ Scuti, $\gamma$ Doradus, roAp, $\beta$ Cephei, Slowly Pulsating B and Be stars, all in the context of solar-like oscillations.
\end{abstract}

Keywords. stars: oscillations, stars: variables: $\delta$ Scuti, stars: variables: $\gamma$ Doradus, stars: variables: Sun-like stars, stars: variables: $\beta$ Cephei, stars: variables: SPB stars, stars: variables: Be stars

\section{Introduction}

There are several mechanisms driving oscillations in pulsating variables. The oldest known is the opacity $(\kappa)$ mechanism acting like a heat engine, converting thermal energy into mechanical energy (e.g. Eddington 1919, Cox 1963). The layers where the thermal energy is stored are the zones connected to (partial) ionisation of abundant elements, which can take place only at specific temperatures. The zone where neutral hydrogen $(\mathrm{H})$ and helium $(\mathrm{He})$ are ionised is at about $14000 \mathrm{~K}$ and close to the surface, whereas the second ionisation zone of He II is at $\sim 50000 \mathrm{~K}$. The driving in the He II ionisation zone is the main source of excitation in stars placed in the classical instability strip such as the $\delta$ Scuti ( $\delta$ Sct) stars. The pulsations of the more massive $\beta$ Cephei ( $\beta$ Cep) and Slowly Pulsating B stars (SPB) are triggered by the $\kappa$ mechanism operating on the iron-group elements (located at $\sim 200000 \mathrm{~K}$ ).

The oscillations in $\gamma$ Doradus stars ( $\gamma$ Dor) are driven by a similar mechanism, in this case, however, it is the bottom of the outer convection zone blocking the flux from the interior (Guzik et al. 2000) and is therefore called convective blocking (see Fig. 1 for exact location in the Hertzsprung-Russell diagram).

Another important mechanism, especially for the present review, is stochastic driving. In stars like the Sun the modes are intrinsically stable, damped by the turbulent convection. Nevertheless the acoustic energy of the convective motion, which is comparable to the sound speed, is sufficient to cause resonance at a star's natural frequencies where a part of the energy is transferred into global oscillation modes. Because of the large number of convective cells the excitation is random, hence stochastic. This is very different from the $\kappa$ mechanism, which excites pulsation coherently. This contrast is a very important way of distinguishing between these two types of driving mechanisms in the signal processing. Another important attribute of stochastic driving is that all the modes in a certain frequency range are excited to observable amplitudes, allowing mode 


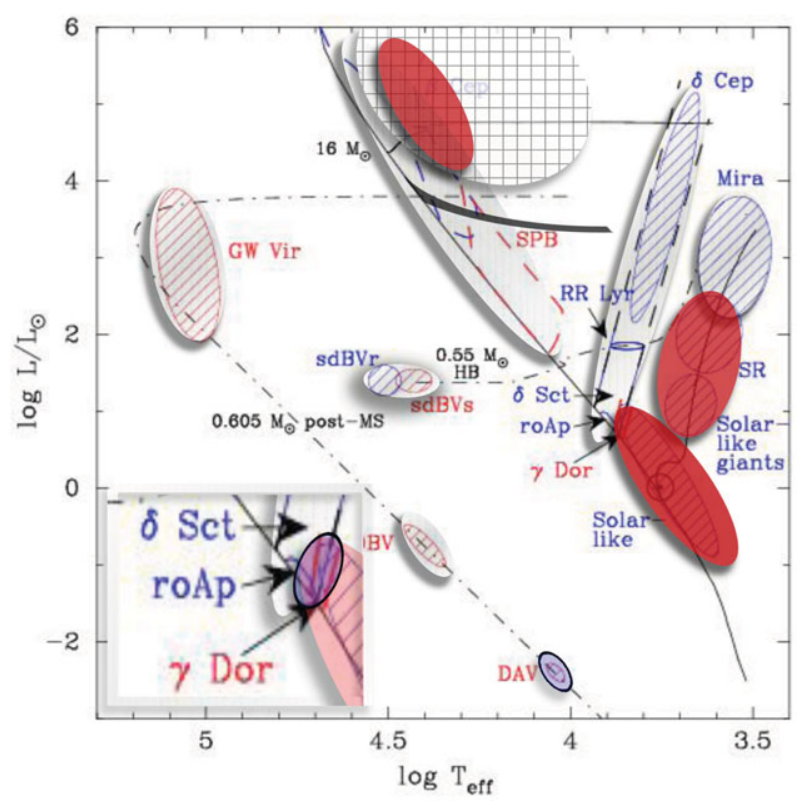

Figure 1. The asteroseismic H-R diagram displays the major types of known pulsating stars. This modified version shows their excitation mechanisms labeled as follows: the $\kappa$ mechanism is depicted in light grey, the stochastic mechanism in dark grey (red in the electronic form) and the grey area surrounded by a black ellipse (blue area in the electronic form) indicates the convective blocking mechanism. The inset is a close-up of the lower part of the classical instability strip. Note the overlap of the different instability strips. The upper hashed region depicts the region where massive stars have a sub-surface convection zone with convective velocities $\geqslant$ $2.5 \mathrm{~km} \mathrm{~s}^{-1}$. The black line, just above the SPB stars shows the division line for surface convection in the Fe ionisation zone. Both regions indicating convection in massive stars are from Cantiello et al. (2009). Adapted from J. Christensen-Dalsgaard and G. Handler.

identification from pattern recognition. This is again in absolute contrast to the heat engine where the mechanism selecting which modes are excited to observable amplitudes is not understood (for a review see Smolec, these proceedings).

However, a star will only pulsate if the conditions inside are just right for a mechanism to work. For the $\kappa$ mechanism, a mode will only be excited if its period of oscillation corresponds to the thermal time scale of the driving zone (e.g. Pamyatnykh 2000), which means that the opacity bump needs to be at a favourable depth in the envelope. Similarly for the convective blocking mechanism, the depth of the convective envelope should be between 3 and $9 \%$ (Guzik et al. 2000). As for the stochastic excitation, what matters is not the depth of the convective layer, but how vigorous the convective motions are.

Stochastically excited pressure modes as observed in the Sun are nearly equally spaced in frequency and give rise to a clear comb-like structure. The almost equidistant spacing in frequency of consecutive modes of the same degree $l$ is the so called large frequency separation $\Delta \nu$ and measures the mean density of a star. The oscillation spectrum in Sun-like stars as well as in red giants is described by an envelope with a frequency of maximum power, for the Sun $\nu_{\max }$ is at $3090 \mu \mathrm{Hz}$ (Huber et al. 2011). The $\nu_{\max }$ and the shape of the oscillation envelope, determined by damping and driving, are related to the acoustic cutoff frequency, $\nu_{\text {ac }} \propto g T_{\text {eff }}^{-1 / 2}$ (e.g. Brown et al. 1991). From that, Kjeldsen \& Bedding (1995) derived the following scaling relation for $\nu_{\max }$ for any other star: $\nu_{\max }=$ $\nu_{\max \odot}\left(M / M_{\odot}\right)\left(R / R_{\odot}\right)^{-2}\left(T_{\text {eff }} / T_{\text {eff } \odot}\right)^{-1 / 2}$. Kjeldsen \& Bedding (1995) also show that $\Delta \nu$, 
when scaled to the Sun, can also be written as $\Delta \nu=\left(M / M_{\odot}\right)^{1 / 2}\left(R / R_{\odot}\right)^{-3 / 2} \Delta \nu_{\odot}$. From observations with the CoRoT and Kepler satellites it was demonstrated that there is a clear empirical relation between $\nu_{\max }$ and $\Delta \nu$ of the form: $\Delta \nu=\alpha\left(\nu_{\max } / \mu \mathrm{Hz}\right)^{\beta}$, where $\alpha$ and $\beta$ can have slightly different values, summarised by e.g. Huber et al. (2011). All these relations are powerful tools to study solar-like oscillators.

The timescale on which a solar-like mode is excited, the mode lifetime, depends on the damping rate. This can be determined by fitting a Lorentzian profile to an observed mode and measuring its line width, where the inverse of the FWHM times $\pi$ gives the mode lifetime. Because the power of the mode is spread over a certain frequency range, the amplitudes and frequencies are also derived by fitting Lorentzian profiles.

Detecting solar-like oscillations in Sun-like stars is easy, however identifying stochastically excited modes in other types of pulsators is complicated and requires a lot of data. In particular discriminating between different excitation mechanisms is sometimes impossible. Temporal variability does not necessarily mean stochastic excitation; it can simply be that there are many closely spaced, unresolved frequencies. The good news is that even in the case where coherent and non-coherent signals are present, one can subtract the coherent peaks without destroying the stochastic signal (Antoci et al. 2013).

Studying the excitation mechanisms of stars with different temperatures, masses and evolutionary stages allows us to determine the structure of stars as well as understand several physical processes. The existence of convective layers plays a major role in the generation of magnetic fields and stellar activity, in the transport of angular momentum, in mixing processes and diffusion, and even in the alignment of planetary systems.

\section{A- and early F-type stars}

The A- and early F-type stars are very complex objects, because the transition from deep and effective to shallow convective envelopes takes place within this spectral region. Figure 2 shows how the outer layers change as a function of temperature for stars located on the zero-age main sequence, from theory (Christensen-Dalsgaard 2000). While the outer $30 \%$ are fully convective for the Sun, it changes dramatically for slightly higher temperatures and separates into the known ionisation zones ( $\mathrm{H}, \mathrm{He} \mathrm{I}$ and $\mathrm{He}$ II). For a late A type star, for example, only the outer $2 \%$ of the radius is still convective (e.g. Kallinger \& Matthews 2009). The right panel of Fig. 2 shows how much of the energy is transported by convection, demonstrating again the drastic change in the convective properties. The grey (electronic version blue) region in the same figure indicates the location where the $\kappa$ mechanism operates in the He II ionisation zone, as assumed to be the case in the $\delta$ Sct stars. Interestingly, Théado et al. (2012) find that if diffusion is implemented in the models, heavy elements will gravitationally settle. Due to the strong accumulation of $\mathrm{Fe}$ in the $\mathrm{Z}$ bump, this region eventually becomes convective, mixing again the material into the higher layers connected by overshooting, hence destroying the steep gradient. Once this layer is homogeneous enough the convection will cease and the cycle repeats. The authors find several convective episodes during the main sequence lifetime of a $2 M_{\odot}$ star.

From an observational point of view, Gray \& Nagel (1989) found from bisector measurements that the granulation boundary crosses the classical instability strip. Also related to the afore-mentioned transition is the so-called rotation boundary. Stars later than F5 have slow rotation rates, on average $v \sin i<10 \mathrm{~km} \mathrm{~s}^{-1}$, with a sharp increase between F0 and F5 (Royer 2009). The measured rotational velocities for the cool stars inside the instability strip are typically higher than $100 \mathrm{kms}^{-1}$. Royer (2009) also reports observational evidence for differential rotation in A type stars, which is related to magnetic 

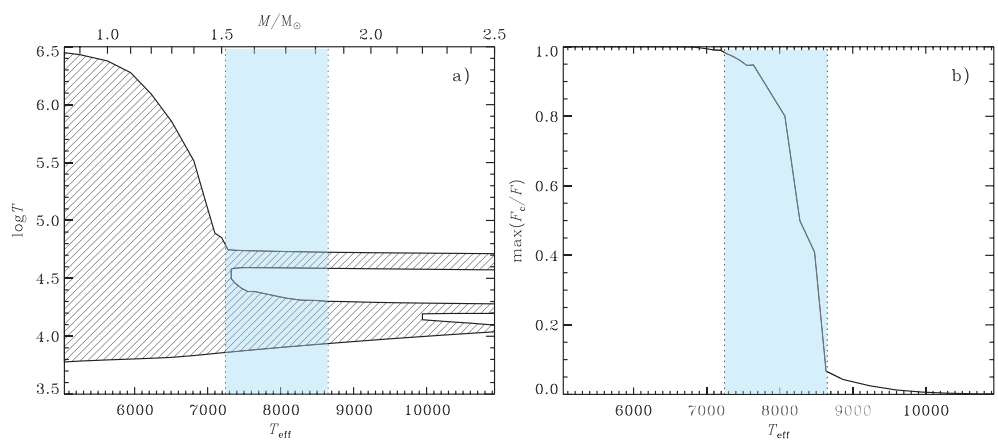

Figure 2. Envelope structure for zero-age main sequence stars as a function of effective temperature (lower abscissa) and mass (upper abscissa). Left: the dashed area indicates the convective regions in the outer layers. It can be seen that the depth of the convective envelope changes dramatically within the classical instability strip (grey area, blue in electronic form). While for stars like the Sun, all three convection zones are combined, for hotter more massive stars the H, He I and He II zones start to separate. Right: $F_{\mathrm{c}} / F$ indicates how much of the energy is transported by convection. Again a clear transition is present within the instability strip. Courtesy of J. Christensen-Dalsgaard.

dynamos like in the Sun. Chromospheric activity disappears only for stars hotter than $8300 \mathrm{~K}$ (Simon et al. 2002), demonstrating that the $\delta$ Sct and $\gamma$ Dor pulsators are still affected by the presence of convective envelopes. Landstreet et al. (2009) and Kallinger \& Matthews (2009) find strong evidence for the signatures of convective motions.

Houdek et al. (1999) and Samadi et al. (2002) predicted that surface convection in the envelopes of $\delta$ Sct stars is still vigorous enough to excite solar-like oscillations. There is no reason why the same prediction should not be generalised for all the stars occupying the same location in the H-R diagram. Solar-like oscillations, if detected, would allow one to perform mode identification from pattern recognition and therefore to also identify the modes of pulsation excited by the $\kappa$ mechanism. As a result we can then reproduce the deep interior of the star by using the $\delta$ Sct oscillations and constrain the overshooting parameter $\alpha_{\mathrm{ov}}$, which is the primary factor to influence the lifetime on the main sequence. The solar-like oscillations on the other hand are most sensitive to outer (convective) envelope, which so far is poorly understood in stars with temperatures higher than $6500 \mathrm{~K}$.

\section{1. (Rapidly oscillating) Ap stars}

Rapidly oscillating (ro)Ap stars are stars with magnetic fields of the order of $\mathrm{kG}$, with periods of oscillations similar to the solar ones (4 to $20 \mathrm{~min}$ ). The regular patterns in the oscillation spectrum of roAp stars are best explained by the oblique pulsator model, where the pulsation axis is inclined with respect to the magnetic and rotation axes (Kurtz 1982, Bigot \& Dziembowski 2002). The excitation mechanism is not entirely understood but is interpreted as the opacity mechanism acting in the $\mathrm{H}$ ionisation zone. In Ap stars the magnetic field suppresses convection at the magnetic poles reducing the damping (Balmforth et al. 2001), triggering the observed p modes. So far there are no solar-like oscillations observed in roAp stars, but also none expected.

\section{2. $\gamma$ Dor stars}

The generally accepted mechanism triggering the high radial order g mode pulsations in $\gamma$ Dor stars is the convective blocking mechanism. Interestingly, the instability domain of the $\gamma$ Dor stars overlaps the one of the $\delta$ Sct stars, suggesting that both types of pulsation 
should be excited simultaneously in stars located there (Fig. 1). Missions like the NASA Kepler satellite established that the $\delta$ Sct and $\gamma$ Dor hybrids are a common phenomenon, rather than an exception (Grigahcène et al. 2010). Intriguingly, from Kepler observations, Uytterhoeven et al. (2011) and Tkachenko et al. (2012) find hybrid pulsators not only in the region where the two instability domains overlap but distributed all over the $\delta$ Sct strip. The convective blocking mechanism, as it is currently understood, cannot operate unless the convective envelope has a certain depth between approximately 3 and $9 \%$ (Guzik et al. 2000); this is not expected to be the case at the observed effective temperatures. This implies that either the hybrids observed at such high $T_{\text {eff }}$ values have still a non-negligible subsurface convection zone or we are missing basic physics. As far as stochastic excitation is concerned, these stars are the perfect targets to search for solar-like oscillations. Nevertheless, up to now, no $\gamma$ Dor/solar-like hybrids were detected, which is very puzzling. The non-detection of stochastic oscillations in these stars becomes even more mysterious when considering the significant number of solar-like oscillators identified within the $\gamma$ Dor instability strip. In other words, there are several $\mathrm{F}$ type stars with high temperatures, higher than some of the $\gamma$ Dor pulsators, showing solarlike oscillations but no g modes. Note that here I consider only targets with reliable temperature determinations (W.J. Chaplin, priv. comm.). To summarise, there are no solar-like oscillations detected in $\gamma$ Dor stars, but from the theoretical point of view we do not understand why. One has to keep in mind, however, that $\gamma$ Dor are usually observed with a sampling cadence not rapid enough to detect high radial-order $\mathrm{p}$ modes.

\section{3. $\delta$ Sct stars}

$\delta$ Sct stars are one of the first known groups of pulsators, yet also one of the least understood. It is generally accepted that the complex pulsational behaviour is the result of the $\kappa$ mechanism acting in the He II ionisation zone. Previously, models predicted more modes to be unstable than observations showed; however, with CoRoT and Kepler, the opposite seems to be the case (e.g. Poretti et al. 2009, García-Hernández et al. 2010, Balona \& Dziembowski 2011, Uytterhoeven et al. 2011). The NASA Kepler spacecraft observed hundreds of $\delta$ Sct stars at a precision where solar-like oscillations, if present, should be detected. One of these stars was HD 187547 (Antoci et al. 2011), at first glance a typical $\delta$ Sct star. However, at high frequencies, the authors detected modes approximately equidistantly spaced, as expected for high radial-order p modes, which are not combination frequencies. The $\kappa$ mechanism, as it is known, cannot excite a continuous frequency region as observed in HD 187547 (Pamyatnykh 2000). Spectroscopic observations exclude that the peaks at high frequencies originate from a possible companion; such a star would be an A- or F-type star and would be visible in the spectrum, which is not. The measured large separation $\Delta \nu$ predicts $\nu_{\max }$ to be exactly where the mode with the highest amplitude in the supposed stochastic region is detected. New data, however showed that the mode lifetimes are longer than the observing run, suggesting coherence over more than 450 days (from scaling relations the mode lifetimes are suggested to decrease with increasing $\left.T_{\text {eff }}\right)$. This is not compatible with the interpretation of 'pure' solar-like oscillations. The word 'pure' in this context should emphasise that there are no studies on how the $\kappa$ mechanism interacts with the stochastic excitation, especially in the case where the periods of pulsations have similar time scales.

Very recent results, in collaboration with Margarida Cunha and Günter Houdek, suggest the presence of a new excitation mechanism for at least the $T_{\text {eff }}$ domain of HD 187547 , i.e. $7500 \mathrm{~K}$. The models, using a non-local and time-dependent treatment of convection (Houdek et al. 1999 and references therein), reproduce the major part of the observed modes in HD 187547, as being excited by the turbulent pressure and not the $\kappa$ mechanism. 
The turbulent pressure can be understood as the dynamical component of convection, associated with the transport of momentum (details will be published in a dedicated article). This proves that convection in the envelope of these stars still plays an important role and can excite pulsations even if not stochastically.

\section{O- and B-type stars}

When it comes to stellar structure, O- and B-type stars are usually described as having a convective core and a purely radiative envelope. Cantiello et al. (2009), however, show that this is not necessarily true (Fig. 1). Even though it might comprise a negligible part of the stellar mass, subsurface convection occurring predominantly in the $\mathrm{Z}$ bump can not only lead to variability in the observed microturbulent velocity but also influence the stellar evolution of massive stars significantly. This is because the photospheric motion caused by convection can strongly influence mass loss by affecting the stellar winds known to exist in these stars (see Cantiello et al. 2009). Furthermore, a convection zone close to the surface can also induce magnetic fields, which may favour mass loss and more importantly, loss of angular momentum.

Samadi et al. (2010) investigated from a theoretical point of view whether the turbulent convection can stochastically excite g modes in massive stars. They find that, while low radial-order g modes might be excited by the convection in the core, the convective envelope is primarily exciting high radial-order g modes. However, the same authors predict the amplitudes to be too low to be observed even with CoRoT or Kepler. Belkacem et al. (2010), on the other hand, find in their exploratory models of a $10 M_{\odot}$ that both the core and the convective outer layers associated with the Fe bump can efficiently drive stochastic oscillations with detectable amplitudes. However, the authors also comment that the convective properties in these temperature domains are poorly understood. Shiode et al. (2013) arrive at a similar conclusion, namely that the convection in the core can stochastically excite g modes to observable amplitudes. They expect the amplitudes to be highest for stars with masses $\geqslant 5 M_{\odot}$. Also from 3D MHD and numerical simulations Browning et al. (2004) and Rogers et al. (2012) find that convection in the core excites internal gravity modes in stars more massive than the Sun. From the observational point of view there is strong evidence for the presence of stochastically excited oscillations in massive O-type stars observed with CoRoT. HD 46149 (O8.5 V) appears to oscillate in stochastic modes fulfilling the scaling laws as expected for solar-type stars and also showing ridges for modes of equal degree but consecutive radial orders as those observed for Sun-like stars (Degroote et al. 2010). The $\kappa$ mechanism is not expected to drive the observed oscillations. Besides that, the temporal variability argues in favour of their stochastic nature. Blomme et al. (2011) find 300 significant frequencies in HD 46966 $(\mathrm{O} 8 \mathrm{~V})$, all displaying temporal variability which might be due to granulation noise. The same authors find similar behaviour in other two $\mathrm{O}$ type stars.

\subsection{SPB stars}

SPB stars pulsate in high radial-order $g$ modes excited by the $\kappa$ mechanism associated with the opacity in the $Z$ bump (Fig. 1). Their instability region overlaps that of the $\beta$ Cep stars, suggesting the presence of both type of pulsations. As in the case of $\gamma$ Dor and $\delta$ Sct stars, observations are consistent with theory (e.g. Handler et al. 2009). According to Cantiello et al. (2009), the only subsurface convective layer present in these stars is in the He II ionisation zone, but it is shallow and inefficient. No solar-like oscillations driven by the convection in the envelope are expected in SPB stars. If there would be any, these would be excited by the convection in the core but none were observed so far. 


\section{2. $\beta$ Cep stars}

The $\beta$ Cep stars pulsate in $\mathrm{p}$ and g modes excited by the $\kappa$ mechanism in the $Z$ bump. From Cantiello et al. (2009) and the theoretical work summarised above, these stars are expected to have a substantial convection layer in the $Z$ bump. Belkacem et al. (2009) reported the detection of solar-like oscillations and opacity driven modes in the $\beta$ Cep star V1449 Aql. The authors argue that the peaks observed at high frequencies are consistent with stochastic oscillations, because of the temporal variability and the detected spacing interpreted as the large frequency separation. Aerts et al. (2011), on the other hand, can seismically reproduce the pulsational behaviour of V1449 Aql without invoking the presence of solar-like oscillations but only $\kappa$ mechanism excitation. They ascribe the power at higher frequencies to non-linear resonant mode coupling between the dominant radial fundamental mode and many other low-order p modes. Degroote (2013) delivers a third possible explanation for the observed frequency spectrum of V1449 Aql, arguing that chaotic behaviour of the very dominant mode can qualitatively reproduce the observed frequency spectrum. As no other $\beta$ Cep pulsators have been found to show solar-like oscillations, the case of V1449 Aql is still a matter of debate.

\subsection{Be stars}

Be stars are stars showing emission in their spectra originating from a circumstellar disk. These can be found spreading over several spectral classes (O, B and early A). So far the Be phenomenon is still not fully understood but can be related to rapid rotation, and meanwhile to pulsations (of SPB and $\beta$ Cep type); the latter were found to be present in all Be stars examined so far (Neiner et al. 2013). Other types of variability attributed to rotation, magnetic fields, stellar winds and outbursts were also observed in these stars (for a complete review on Be stars see Porter \& Rivinius 2003). A very fortunate case is the Be star HD 49330 (B0IVe) which was observed during an outburst with CoRoT as well as from ground (Huat et al. 2009). In this star the authors find a clear correlation between the outburst and the presence of g modes. While during the quiescent phase the pulsations are predominantly in the p-mode regime, during the outburst the g modes are enhanced to higher amplitudes and are also very unstable indicating short mode lifetimes. The modelling of this star, even considering rapid rotation, shows that the $\kappa$ mechanism cannot excite the observed g modes. A very recent study of another Be star (HD 51452, Neiner et al. 2012) suggests that the g modes observed in the two afore-mentioned Be stars are in fact stochastically driven gravito-inertial modes. These oscillations are excited by the convection in the core and enhanced to observable amplitudes by the rapid rotation (see Neiner et al. 2012 and Mathis et al. 2013 for details).

\section{Conclusions}

The potential to detect stochastically excited oscillations in stars significantly more massive than the Sun became possible only with the space missions CoRoT and Kepler. It is not only the quality of the data which is outstanding, but also the uninterrupted and long observing seasons which demonstrated that we need to revisit our knowledge on pulsation mechanisms (Fig. 1). Convection is one of the most complex processes in astrophysics and therefore often neglected with the argument that convection zones in stars hotter than early $\mathrm{F}$ are negligible. However, the latest results reviewed in this article demonstrate that the impact of convection needs to be taken into account.

\section{Acknowledgements}

Funding for the Stellar Astrophysics Centre (SAC) is provided by The Danish National Research Foundation. The research is supported by the ASTERISK project 
(ASTERoseismic Investigations with SONG and Kepler) funded by the European Research Council (Grant agreement no.: 267864).

\section{References}

Aerts, C., Briquet, M., Degroote, P., Thoul, A., \& van Hoolst, T. 2011, A\& A, 534, A98

Antoci, V., Handler, G., Campante, T. L., et al. 2011, Nature, 477, 570

Antoci, V., Handler, G., Grundahl, F., et al. 2013, MNRAS, 435, 1563

Balmforth N. J., Cunha, M. S., Dolez, N., Gough, D. O., \& Vauclair, S. 2001, MNRAS, 323, 362

Balona, L. A. \& Dziembowski, W. A. 2011, MNRAS, 417, 591

Belkacem, K., Samadi, R., Goupil, M.-J., et al. 2009, Science, 324, 1540

Belkacem, K., Dupret, M.-A., \& Noels, A. 2010, A\& $A$, 510, A6

Bigot, L. \& Dziembowski, W. A. 2002, A\&A, 391, 235

Blomme, R., Mahy, L., Catala, C., et al. 2011, AछA, 533, A4

Brown, T. M., Gilliland, R. L., Noyes, R. W., \& Ramsey, L. W. 1991, ApJ, 368, 599

Browning, M. K., Brun, A. S., \& Toomre, J. 2004, in: J. Zverko, J. Žižňovský, S. J. Adelman, \& W. W. Weiss (eds.), The A-Star Puzzle, Proc. IAU Symposium No. 224 (Cambridge: Cambridge University Press), p. 149

Cantiello, M., Langer, N., Brott, I., et al. 2009, A\&SA, 499, 279

Cox, J. P. 1963, ApJ, 138, 487

Christensen-Dalsgaard, J. 2000, ASP-CS, 210, 454

Degroote, P. 2013, MNRAS, 431, 255

Degroote, P., Briquet, M., Auvergne, M., et al. 2010, A\& A, 519, A38

Eddington, A. S. 1919, MNRAS, 79, 177

García Hernández, A., Moya, A., Michel, E., et al. 2009, A\&3A, 506, 79

Grigahcène, A., Antoci, V., Balona, L. A., et al. 2010, ApJ, 713, L192

Guzik, J. A., Kaye, A. B., Bradley, P. A., Cox, A. N., \& Neuforge, C. 2000, ApJ, 542, L57

Gray, D. F. \& Nagel, T. 1989, ApJ, 341, 421

Handler, G., Matthews, J. M., Eaton, J. A., et al. 2009, ApJ, 698, 56

Houdek, G., Balmforth, N. J., Christensen-Dalsgaard, J., \& Gough, D. O. 1999, A\&A, 351, 582

Huat, A.-L., Hubert, A.-M., Baudin, F., et al. 2009, A\& A, 506, 95

Huber, D., Bedding, T. R., Stello, D., et al. 2011, ApJ, 743, 143

Kallinger, T. \& Matthews, J. M. 2010, ApJ, 711, L35

Kjeldsen, H. \& Bedding, T. R. 1995, A\& A, 293, 87

Kurtz, D. W. 1982, MNRAS, 200, 807

Landstreet, J. D., Kupka, F., Ford, H. A., et al. 2009, A\&A A, 503, 973

Mathis, S., et al., 2013, A\&GA, in press

Neiner, C., Floquet, M., Samadi, R., et al. 2012, A\&A, 546, A47

Neiner, C., et al. 2013, H. Shibahashi and A. E. Lynas-Gray (eds.), Progress in Physics of the Sun and Stars: A New Era in Helio- and Asteroseismology, ASP-CS, in press

Pamyatnykh, A. A. 2000, ASP-CS, 210, 215

Poretti, E., Michel, E., Garrido, R., et al. 2009, A\&A, 506, 85

Porter, J. M. \& Rivinius, T. 2003, PASP, 115, 1153

Rogers, T. M., Lin, D. N. C., \& Lau, H. H. B. 2012, ApJ, 758, 6

Royer, F. 2009, Lecture Notes in Physics, 765, 207

Samadi, R., Goupil, M.-J., \& Houdek, G. 2002, A\&BA, 395, 563

Samadi, R., Belkacem, K., Goupil, M.-J., Dupret, M.-A., Brun, A. S., \& Noels, A. 2010, ApESSS, 328,253

Shiode, J. H., Quataert, E., Cantiello, M., \& Bildsten, L. 2013, MNRAS, 430, 1736

Simon, T., Ayres, T. R., Redfield, S., \& Linsky, J. L. 2002, ApJ, 579, 800

Théado, S., Alecian, G., LeBlanc, F., \& Vauclair, S. 2012, A\&A, 546, A100

Tkachenko, A., Lehmann, H., Smalley, B., Debosscher, J., \& Aerts, C. 2012, MNRAS, 422, 2960

Uytterhoeven, K., Moya, A., Grigahcène, A., et al. 2011, A\&A, 534, A125 